\title{
Relative Abundance of Potato Psyllid Haplotypes in Southern Idaho Potato Fields During 2012 to 2015, and Incidence of 'Candidatus Liberibacter solanacearum' Causing Zebra Chip Disease
}

Jennifer Dahan, Department of Plant, Soil and Entomological Sciences (PSES), University of Idaho, Moscow 83844; Erik J. Wenninger, Department of PSES and Kimberly Research and Extension Center, University of Idaho, Kimberly 83341; Brandon Thompson and Sahar Eid, Department of PSES, University of Idaho, Moscow 83844; Nora Olsen, Department of PSES and Kimberly Research and Extension Center, University of Idaho, Kimberly 83341; and Alexander V. Karasev, Department of PSES, University of Idaho, Moscow 83844

\begin{abstract}
Zebra chip (ZC) disease, a serious threat to the potato industry, is caused by the bacterium 'Candidatus Liberibacter solanacearum' (Lso). Five haplotypes (hapA to hapE) of this pathogen have been described so far in different crops, with only hapA and hapB being associated with ZC in potato. Both haplotypes are vectored and transmitted to a variety of solanaceaeous plants by the tomato/potato psyllid, Bactericera cockerelli (Šulc). Psyllids are native to North America, and four haplotypes have been identified and named based on their predominant geographic association: Northwestern, Central, Western, and Southwestern. Although all psyllid haplotypes have been found in southern Idaho potato fields, data on relative haplotype abundances and dynamic changes in the fields over time have not previously been reported.

Here, psyllid samples collected in Idaho potato fields from 2012 to 2015 were used to clarify spatial and temporal patterns in distribution and abundance of psyllid and Lso haplotypes. A shift from hapA toward hapB population of Lso was revealed during these four seasons, indicating possible evolution of Lso in Idaho fields. Although we confirmed that Western psyllids were the most abundant by far during the four seasons of observation, we also observed changes in abundance of other haplotypes, including increased diversity of psyllid haplotypes during 2015. Seasonal changes observed for the Northwestern and Central haplotypes could potentially be linked to psyllid migration and/or habitat changes. South-central Idaho exhibited more diversity in psyllid haplotypes than southwestern Idaho.
\end{abstract}

Zebra chip (ZC) disease is an emerging and potentially devastating disease to the U.S. potato industry. First described in Mexico in 1994 (Secor and Rivera 2004), it expanded to Central America and the United States (Munyaneza et al. 2009), reaching the Pacific Northwest states of Idaho, Washington, and Oregon in 2011 (Crosslin et al. 2012a, 2012b; Munyaneza et al. 2009). ZC is caused by a bacterium, 'Candidatus Liberibacter solanacearum' (Lso) (Hansen et al. 2008; Liefting et al. 2009). It is an unculturable $\alpha 2$-proteobacterium, which is phloem-restricted and associated with a diversity of symptoms ranging from stunting, wilting, leaf rolling, and/or purpling, and ultimately plant death (reviewed in Munyaneza 2012). The most diagnostic visual symptom of this disease is the appearance of dark stripes in infected tubers (especially pronounced when fried), which alters flavor quality and makes fried products derived from those tubers unmarketable. The potentially devastating economic effects of ZC to the potato industry in the Pacific Northwest has driven extensive efforts to monitor potato psyllids and Lso throughout the potato-producing areas of this region (Wenninger et al. 2013).

To date, five haplotypes of Lso, designated hapA, hapB, hapC, hapD, and hapE, have been described based on single nucleotide polymorphisms (SNPs) in the 16S rRNA, 16S/23S ISR, and 50S rplJ and rplL ribosomal protein genes (Nelson et al. 2011, 2013). Several molecular methods have been developed to allow for discrimination among haplotypes (Ananthakrishnan et al. 2013; Wen et al. 2011; Wen et al. 2013). The different haplotypes exhibit a diversity in host range and insect vector specificity. Haplotypes $C$ to $E$ have been described in Europe and Africa in association with celery and carrot diseases, whereas haplotypes A and B are associated with $\mathrm{ZC}$ in potato

Corresponding author: A. V. Karasev; E-mail: akarasev@uidaho.edu

*The $\boldsymbol{e}$-Xtra logo stands for "electronic extra" and indicates that one supplementary figure and two supplementary tables are published online.

Accepted for publication 20 December 2016.

C 2017 The American Phytopathological Society and other solanaceaeous crops (Loiseau et al. 2014; Munyaneza et al. 2010, 2015; Murphy et al. 2014; Nelson et al. 2013; Tahzima et al. 2014; Teresani et al. 2014; Thinakaran et al. 2015b). To date, hapA Lso has been found in potato crops from Central America to the western United States through western Mexico, and also in potato and tomato in New Zealand. This includes the Pacific Northwest potato fields, where the ZC presence in 2011 and 2012 was linked to hapA Lso only (Swisher et al. 2014). HapB Lso has been described in potato in eastern Mexico and the central United States. Overlap between the two haplotypes has been observed in Texas, Nebraska, and Arkansas. In the areas where the two haplotypes co-occur, a shift from dominance in hapB to dominance in hapA has recently been observed, indicating that the Lso population in the field can be dynamic (Wen et al. 2013). This has raised concerns about evolution of the Lso populations in the Pacific Northwest over time and space. No study has yet reported clear biological differences between hapA and hapB, although ZC disease symptoms in New Zealand, where only hapA has been found, were seemingly less severe than what was observed in Texas, where hapB used to be predominant. This suggested that hapB could be more virulent than hapA (Wen et al. 2013). Although comparative genomic analysis between hapA and hapB showed genetic complexity within this bacterial species, no obvious genetic factors were observed that might explain Lso population shifts observed in the field (Thompson et al. 2015).

Infection of potato plants by hapA and hapB Lso occurs via its insect vector, the tomato/potato psyllid, Bactericera cockerelli (Šulc) (Hemiptera: Triozidae). This insect exhibits complete development on a wide range of solanaceous hosts, including potato. Endemic to the western United States and Central America, four haplotypes of potato psyllids have been described, based on the mitochondrial CO1 pseudogene (Chapman et al. 2012; Swisher et al. 2012). Each haplotype exhibits a different predominant, although not mutually exclusive, geographical association and has been named accordingly: (i) Central, present in Central America and central United States; (ii) Western, dominating the western part of the United States; (iii) Northwestern, only found in Washington, Oregon, and Idaho; and (iv) Southwestern, originally described in New Mexico and Colorado (Liu et al. 2006; Swisher et al. 2012, 2013a, 2013b, 2014). All life stages of psyllids can acquire Lso by feeding on infected plants, and 
the bacterium can be transovarially transmitted (Hansen et al. 2008). So far, no study has demonstrated any physiological differences in behavior or Lso transmission among psyllid haplotypes (Mustafa et al. 2015a, 2015b, 2015c; Thinakaran et al. 2015a, 2015c).

Although psyllid presence in the southern and central United States has been well documented, only recently have they been shown to infest the Pacific Northwest, during potato growing seasons (Munyaneza et al. 2009; for comprehensive reviews, see Butler and Trumble 2012; Horton et al. 2015). Following the ZC disease outbreak in Idaho, Washington, and Oregon in 2011, attention focused on psyllid populations occurring in the Pacific Northwest. Early haplotyping analyses in Washington and Oregon showed that Lso infection might have been associated with Western psyllids coming northward from southern regions (Swisher et al. 2014), while Northwestern psyllids, found free of Lso at that time, were thought to be a permanent resident of the Pacific Northwest (Swisher et al. 2013b). Seasonal movement of psyllids, as well as their ability to overwinter in the northwestern part of the United States, have long been a subject of studies (Murphy et al. 2013, 2014). Since the discovery that Solanum dulcamara (bittersweet nightshade), in that area, can serve as an alternative wild host plant, Northwestern psyllids have been found to be able to overwinter on that plant. This makes bittersweet nightshade a potential and putative bridge between potato growing seasons, along with other known alternative host plants [e.g., Lycium spp. and Convolvulus spp. (Swisher et al. 2013b; Thinakaran et al. 2015b)]. It appears that psyllid infestation in the Pacific Northwest is probably under diverse constraints, and highly dynamic.

As a direct result of difficulties to decipher origin(s) and occurrence of psyllids in the Pacific Northwest, ZC epidemiology is not yet fully understood. While a correlation between capture of Lso-positive psyllids and detection of infected plants and/or tubers in the fields has been observed (Goolsby et al. 2012), Lso has also been observed persisting over seasons at a very low level in psyllids population while no infected potato plants could be found in the field (personal observation). This further complicates the prediction of disease outbreaks, although maintaining a low level of psyllids in the fields by spraying helps keep ZC at bay (Goolsby et al. 2012).

To gain insights into the psyllid population dynamics in Idaho potato fields and to help in clarifying the epidemiology of ZC linked with psyllids, we made use of an ongoing monitoring program aimed at sampling potato psyllids and Lso across southern Idaho potato fields over the growing seasons from 2012 through 2015. All psyllids collected were systematically screened for Lso, and Lso-positive and Lso-negative psyllids were haplotyped to evaluate seasonal dynamics in abundance of different haplotypes and interactions with Lso incidence. Concomitantly, haplotypes of Lso present in positive samples were assessed through the development of a cleaved amplified polymorphic sequences (CAPS) marker to clarify any relationships between psyllid haplotype and Lso haplotype. Furthermore, we established seasonal patterns of changes in prevalence of the Lso and psyllid haplotypes present in Idaho potato during the four seasons of observation, 2012 to 2015.

\section{Materials and Methods}

Origin of psyllid samples used in this study. Psyllids were collected in southern Idaho potato fields through the University of Idaho psyllid monitoring survey which started in May 2012, according to the method previously described (Wenninger et al. 2013). Briefly, sticky traps were deployed on the edge of $\sim 100$ commercial potato fields, from mid May through vine kill in September. They were set up to cover the potato-producing area along the Snake River Basin. This area comprised the so-called Treasure Valley (southwestern Idaho) and Magic Valley (south-central Idaho; Fig. 1). Each sticky trap was replaced every week, and psyllids captured on collected traps were counted and recovered by scraping them off the sticky card for Lso testing and haplotyping as described below. It is worth mentioning that psyllids cannot be recovered fully following that method, meaning that samples consisted of body parts of various sizes. Samples analyzed also included post-season samples and samples submitted by growers.

A small subset of the 2012 samples (144 psyllids, including 67 Lso-positive samples) was partially analyzed for psyllid haplotypes and reported previously (Swisher et al. 2014); these samples were not analyzed or presented in this work. Here, a larger, distinct set of 558 psyllids, including 304 Lso-positive ones, collected in 2012 was analyzed for psyllid haplotypes and also for Lso haplotypes along the samples collected in three other seasons, 2013 to 2015. Overall, 1,457 psyllids were haplotyped and sorted by the year of collection (Table 1). In order to evaluate the population structure of Lso in Lso-positive samples, all 269 of these samples were haplotyped.

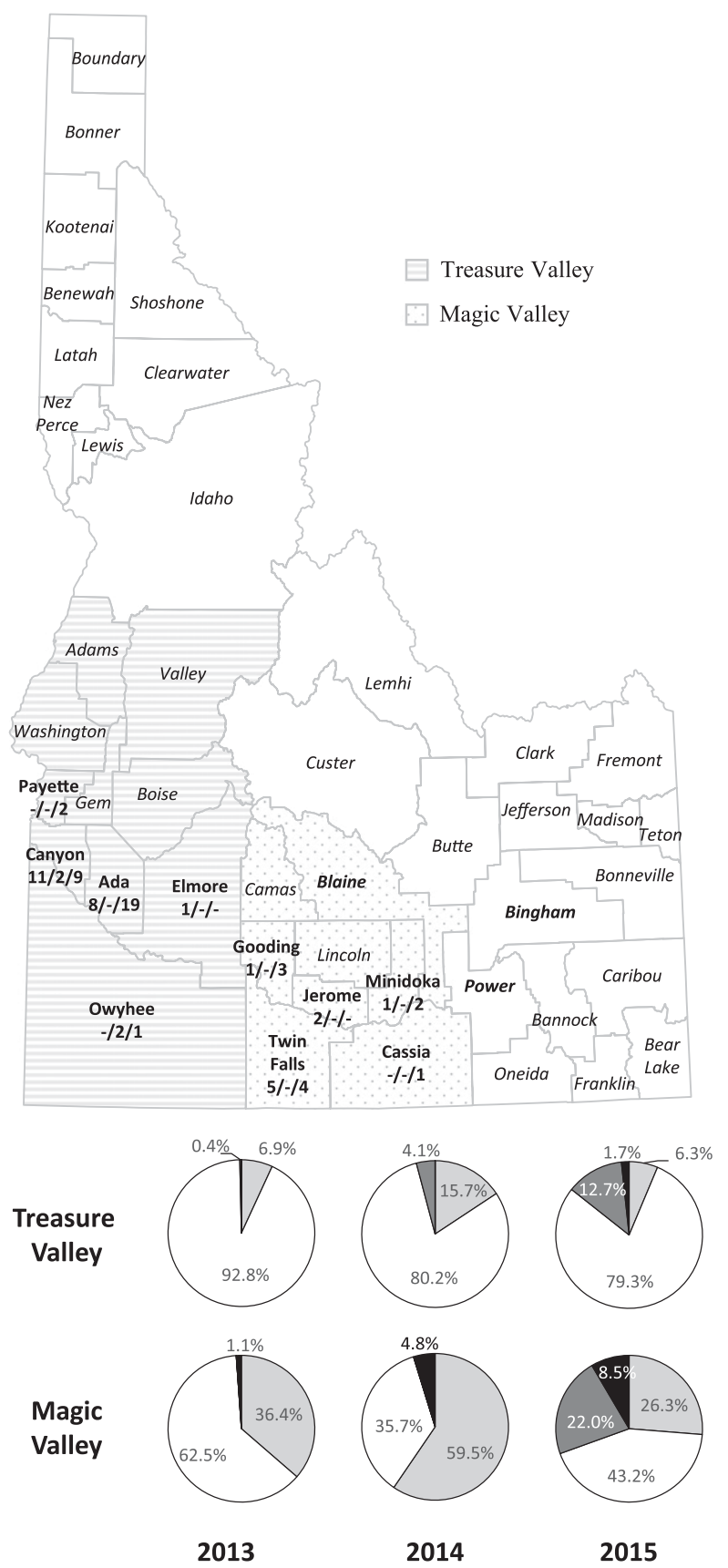

Fig. 1. Relative abundance of potato psyllid haplotypes separated by geographic region. The relative abundance of each psyllid haplotype, obtained by High Resolution Melting analysis, was analyzed based on the county each psyllid was collected from. The areas considered were the Magic Valley and the Treasure Valley, which are represented in the Idaho map as indicated in the key. For each area, monitored counties are in bold letters. Relative abundances of psyllid haplotypes in each area and for each considered year are given in the pie charts (white, Western haplotype; light grey, Northwestern haplotype; dark grey, Central haplotype; black, Southwestern haplotype). Numbers of positive psyllids collected each potato season in 2013,2014 , and 2015 , respectively, in a sampled county are indicated under the name of the county; '-', no positive psyllid collected that year. 
Psyllid DNA extraction. DNA were extracted from psyllids by the CTAB method described in (Crosslin et al. 2011), modified as followed. Briefly, individual psyllids stored in ethanol were dried on paper and resuspended in $300 \mathrm{ul}$ of CTAB buffer (2\% hexadecyltrimethylammoniumbromide (CTAB), $1.4 \mathrm{M} \mathrm{NaCl}, 0.2 \%$ 2-mercaptoethanol, $20 \mathrm{mM}$ EDTA, $100 \mathrm{mM}$ Tris-HCl, $\mathrm{pH}$ 8.0). After grinding using a micropestle, samples were heated at $65^{\circ} \mathrm{C}$ for $15 \mathrm{~min}$. Halfvolume of chloroform/isoamylic alcohol (24:1, v:v) was added and after mixing the tubes were centrifuged $10 \mathrm{~min}$ at $16000 \mathrm{~g}$ at $4{ }^{\circ} \mathrm{C}$. Aqueous phase was transferred to a tube containing $5 \mu \mathrm{g}$ glycogen and $25 \mu \mathrm{l}$ sodium acetate $3 \mathrm{M}, \mathrm{pH}$ 5.2. After addition of $600 \mu \mathrm{l}$ absolute ethanol, tubes were stored at $-20^{\circ} \mathrm{C}$ for at least $1 \mathrm{~h}$ and up to overnight. Precipitated DNA was then pelleted by centrifugation at $20000 \mathrm{~g}$ for $15 \mathrm{~min}, 4^{\circ} \mathrm{C}$ and washed with $500 \mu 170 \%$ ethanol. After drying, DNA was resuspended in $35 \mu \mathrm{l}$ of di-deionized water and stored at $-20^{\circ} \mathrm{C}$ for later uses.

Lso testing. To check for the presence of Lso bacteria in the collected psyllids, two conventional PCR amplifications were carried out on each extracted DNA sample following previously described methods (Crosslin et al. 2011). The two primer pairs used were OA2/OI2c, targeting the bacterial ribosomal $16 \mathrm{~S}$ gene, and OMBF/OMBR, targeting an outer membrane protein gene. The resulting PCR products were analyzed on agarose gels stained with ethidium bromide. Samples were considered positive for Lso if amplification was observed for at least one of the primer pairs. Known Lso-positive samples were used as positive controls along every PCR run.

Lso haplotyping. To haplotype the bacteria present in Lsopositive samples, a CAPS marker approach was developed, based on a particular SNP in the 16S-23S intergenic spacer region. Fragments were amplified from the Lso positive samples, using primers able to hybridize to both hapA and hapB (LsoCAPS_F, 5'-CGTTGTA TAACCGCACGTTG-3' and LsoCAPS_R, 5'-TGTACGGGGCTAT CACCTTC-3'). After amplification, PCR products were subjected to digestion by the restriction enzyme BslI. The digestion products were then analyzed on agarose gels. If needed, a nested PCR was performed on Lso-positive samples that did not give any amplification during the first PCR, using a 3'-nested primer (LsoCAPS_R2, 5'-CGTTCGCTCG CCACTACTAA-3') and providing a fragment still containing the divergent BsII restriction site. The resulting PCR products were also digested as described above.

Psyllid haplotyping. All Lso-positive psyllids and a subset of randomly selected Lso-negative psyllids were haplotyped, following the procedure described by Swisher et al. (2012, 2013a). The HRM analysis was conducted on a Roche Lightcycler 96 and its associated software (Bâle, Switzerland). The primer pairs CO1 F3/CO1 meltR and $\mathrm{CO} 1$ meltF/CO1 meltR were used in the present study, which allowed the haplotyping of all four described potato psyllid haplotypes. Cloned PCR fragments corresponding to the four known haplotypes, as checked by sequencing, were used as positive controls for each primer pair.

Cumulative degree-day analysis. Temperature data were obtained from weather stations maintained by the U.S. Bureau of Reclamation's AgriMet network (https://www.usbr.gov/pn/agrimet/) and the National Centers for Environmental Information (https://www. ncdc.noaa.gov). For the purpose of this study, degree day accumulated for the monitored region for each year was calculated using the sum of the maximum and minimum temperature for a given date divided by two and subtracting from that figure the lower developmental threshold temperature of the potato psyllid $\left(7.1^{\circ} \mathrm{C}\right)$ (Tran et al. 2012). Weekly sample dates for each site were converted to cumulative degree days for that week, using a biofix date of 1 January for that year, and the cumulative value was averaged across sites at the end of each month.

\section{Results}

Psyllid and Lso haplotyping were conducted on psyllid samples collected in southern Idaho between June 2012 and October 2015 (see Table 1). The total number of psyllids tested for Lso exceeded 3,500, with 378 found Lso-positive. More than 1,000 psyllids were tested in each of three seasons $(2012,2013$, and 2015), whereas during the 2014 season far fewer psyllids were captured. In 2012, incidence of the Zebra chip disease in the Idaho fields was $\sim 1 \%$ overall, while several fields in Magic Valley had ZC incidence above 15\%, and symptomatic plants were observed in these affected fields as early as July of 2012 (Wenninger, Olsen, and Karasev, unpublished data). The 2012 was the year when the Lso incidence in the psyllids was the highest during the entire observation period, between 2012 and 2015. No or very few plants across the growing regions exhibiting symptoms were spotted the following years, while Lso was still present but at a rather low prevalence.

Development of a CAPS marker to distinguish hapA and hapB Lso. In order to assess the population of Lso bacteria infecting Idaho potato psyllids, a CAPS marker in the 16S-23S intergenic spacer region, based on those SNPs, was developed (Fig. 2). In particular, one of these SNPs creates a digestion site for the restriction enzyme BslI in the hapA sequence. Primers were designed to amplify a 500-bp fragment overlapping a common BsII digestion site in hapA and hapB sequences and the additional one created by a SNP in the hapA sequence. The Lso hapA fragment then contained two sites for BslI, and the Lso hapB sequence had only one. Consequently, the digestion of the PCR product by the restriction enzyme gave two fragments when amplified from hapB Lso, and three fragments when amplified from hapA (Fig. 3). Sequencing confirmed the haplotyping using this CAPS marker, making this method a fast, inexpensive, and reliable one to haplotype Lso in psyllid samples.

Haplotyping of Lso in Idaho fields between 2012 and 2015. The Lso haplotyping technique developed was applied to Lso-positive psyllid samples from the 2012 through 2015 seasons (Table 1). Interestingly, not all samples could be amplified, suggesting either a very low titer of Lso in those psyllids or instability of the bacterial DNA once extracted.

In 2012, all of the 224 amplified samples (out of 304 Lso-positives) contained hapA Lso. In 2013, while the majority of the haplotyped Lso-positive samples were hapA, two samples out of 15 were found to represent hapB. Although the number of positive samples was extremely low in 2014, hapB was still present in the field (one sample out of three haplotyped, from a total of four Lso-positives). Finally, in 2015, the proportion of hapB samples increased dramatically, reaching $84 \%$ of the samples that could be amplified. In addition, two mixed samples were also detected. It is interesting to note that sequencing of the PCR fragment used for CAPS analysis did not show any divergence from the reference sequences present in databanks for each haplotypes in tested samples. Geographically, hapA and hapB samples were found in both the Magic and Treasure valleys, whereas mixed hapA/hapB samples were found only in the Treasure Valley (Canyon County; Fig. 1).

Psyllid populations in the field. Haplotyping of psyllids was carried out whenever possible on all Lso-positive psyllids as well as on a

Table 1. Summary of the survey of psyllids collected in Southern Idaho for each year from 2012 to 2015. The total number of psyllids collected, number of Lso-positive psyllids, abundance of psyllid haplotypes, and Lso haplotypes are shown for each year

\begin{tabular}{|c|c|c|c|c|c|c|c|}
\hline & \multirow[b]{2}{*}{ Total } & \multirow[b]{2}{*}{ Psyllids haplotyped } & \multirow[b]{2}{*}{ Lso-positive psyllids } & \multirow[b]{2}{*}{ Lso haplotyped } & \multicolumn{3}{|c|}{ Lso haplotypes } \\
\hline & & & & & $\mathbf{A}$ & B & Mixed A/B \\
\hline 2012 & 1150 & 558 & $304(26.4 \%)$ & 224 & 224 & 0 & 0 \\
\hline 2013 & 1084 & 369 & $29(2.7 \%)$ & 15 & 13 & 2 & 0 \\
\hline 2014 & 170 & 164 & $4(2.4 \%)$ & 3 & 2 & 1 & 0 \\
\hline 2015 & 1134 & 358 & $40(3.5 \%)$ & 27 & 4 & 21 & 2 \\
\hline
\end{tabular}


subset of Lso-negative psyllids collected over the four years of this study, except during 2014, where nearly all psyllids were haplotyped because of the low number of psyllids collected. Overall, haplotyping revealed that similar to 2012 (Swisher et al. 2014), the predominant haplotype in the Idaho potato fields during each year was the Western one (Fig. 4). The Western psyllids represented $85.6 \%$ of all haplotyped samples in 2013, but this proportion decreased to $68.3 \%$ and $66.1 \%$ in 2014 and 2015, respectively. This decrease reflected an increase in the relative abundance of the Northwestern and Central haplotypes. The Northwestern haplotype exhibited low incidence during $2012(4.5 \%)$, but increased in relative abundance during 2013 (13.8\%) and 2014 (27.4\%) before dropping to $12.7 \%$ in 2015 . The Central haplotype exhibited a different dynamic. Whereas Central psyllids were barely present in 2013, representing only $0.27 \%$ of the total haplotyped population, they increased in proportion in $2014(3 \%)$ and reached $15.4 \%$ in 2015 . Finally, the Southwestern haplotype, which exhibited only a $0.27 \%$ incidence in 2013 , increased in $2014(1.2 \%)$ to reach $4.1 \%$ in 2015.

Among the Lso-positive psyllids, the relative abundance of the different haplotypes exhibited a distribution pattern that was similar to the whole population studied (Fig. 4). One exception was that no Southwestern psyllid carrying Lso was found in any year. Another exception was 2014, in which no Northwestern psyllid was observed to be carrying the bacterium; however, this might have been due to the very low number of Lso-positive psyllids that season (only four), which limits conclusions that may be drawn for this year.

Dynamics of psyllid populations during the potato growing season. The relative abundance of each psyllid haplotype (Lso-negative and Lsopositive psyllids pooled) was plotted by cumulative degree days, with time point corresponding to the end of each collection month from June to September (Fig. 5; Supplementary Table S1). October was not included in this analysis given the low number of psyllids collected during that month. Psyllid population dynamics changed over time. For the Northwestern haplotype, a consistent pattern was observed among all four years, independent of the relative abundance of this haplotype. Northwestern psyllids were the most prevalent in June and July, except in 2012 when they were at a low level in June. Their proportion then decreased in August and September in all years. For
Central psyllids, a reverse trend was observed relative to the Northwestern haplotype, at least during the two years they were more abundant (2012 and 2015). Indeed, Central psyllids were present at a low level in June of 2012 and 2015, but their abundance gradually increased through September. The representation of Western psyllids exhibited no consistent pattern in relative abundance from year to year, seemingly depending on the balance with the Northwestern and Central haplotypes and changing accordingly to the shifts observed for these populations. Finally, abundance of Southwestern psyllids was too low in any of these four years to detect any seasonal variations. It is worth noting that the population of psyllids present in June showed

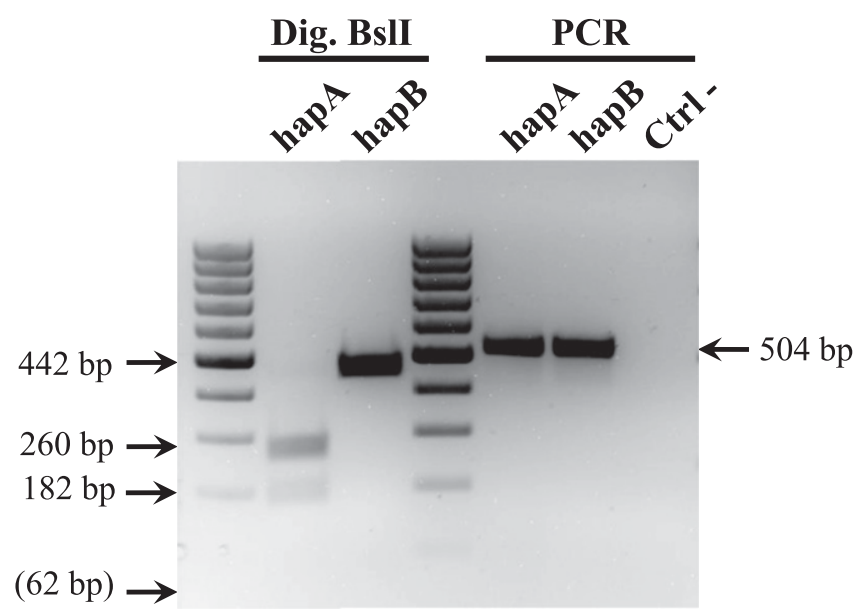

Fig. 3. Analysis of the amplification products and digestion fragments used for Lso haplotyping. A 504-bp fragment located in the 16S-23S ISR of Lso was amplified from the 2 Lso haplotypes A (hapA) and B (hapB). The resulting PCR products were digested by the restriction enzyme Bsll. Both PCR products and digestion products were subjected to electrophoresis on an agarose gel stained with ethidium bromide, along with a molecular weight marker and a negative control (Ctrl -) for PCR. Sizes of the different fragments observed are indicated. The smallest fragment obtained after digestion was not visible, and its length is indicated between parentheses.

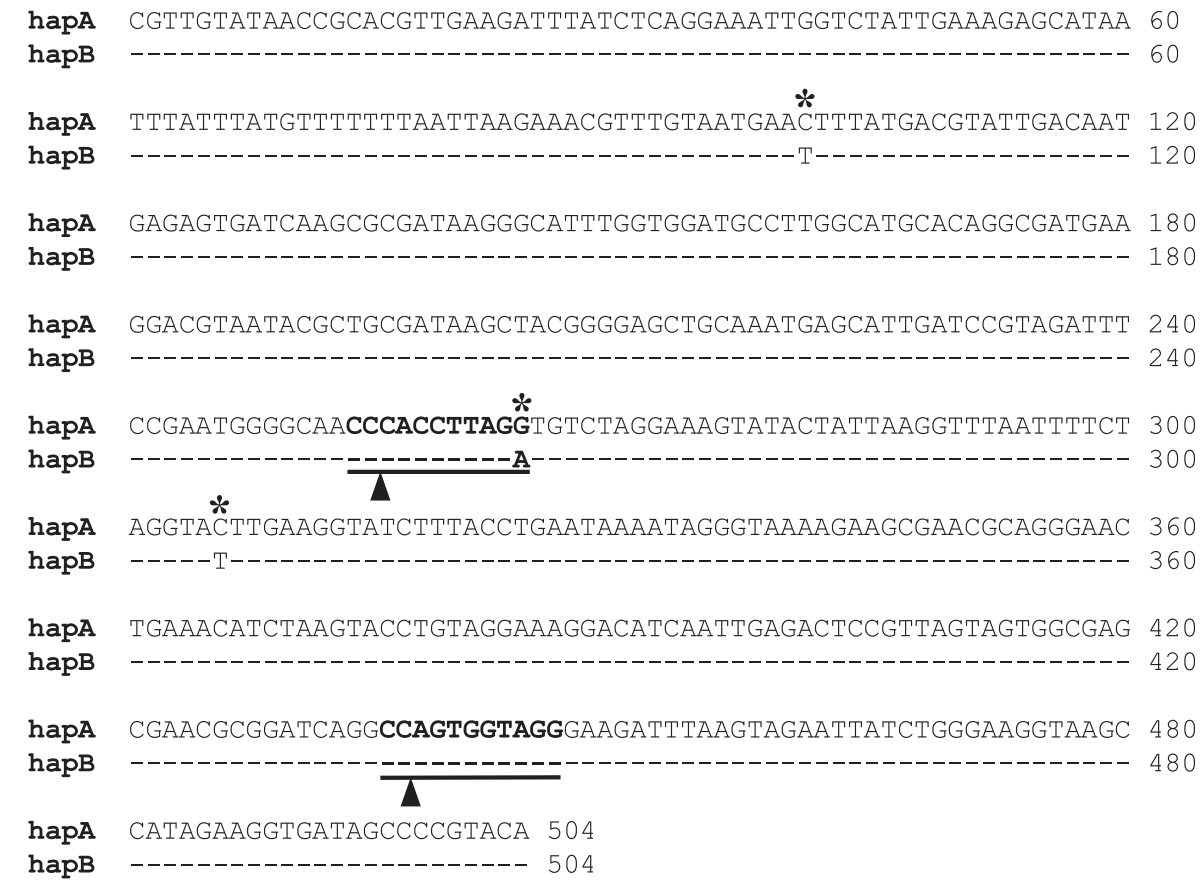

Fig. 2. Alignment of the hapA and hapB 16S-23S intergenic spacer region fragments amplified for Lso haplotyping. The alignment of the 16S-23S fragment amplified from Lso hapA and Lso hapB used for the CAPS marker analysis showed several SNPs between the 2 haplotypes, indicated by the asterisk. The restriction sites for the Bsll restriction enzyme are in bold characters and underlined. The cutting site is indicated by a black arrow. 


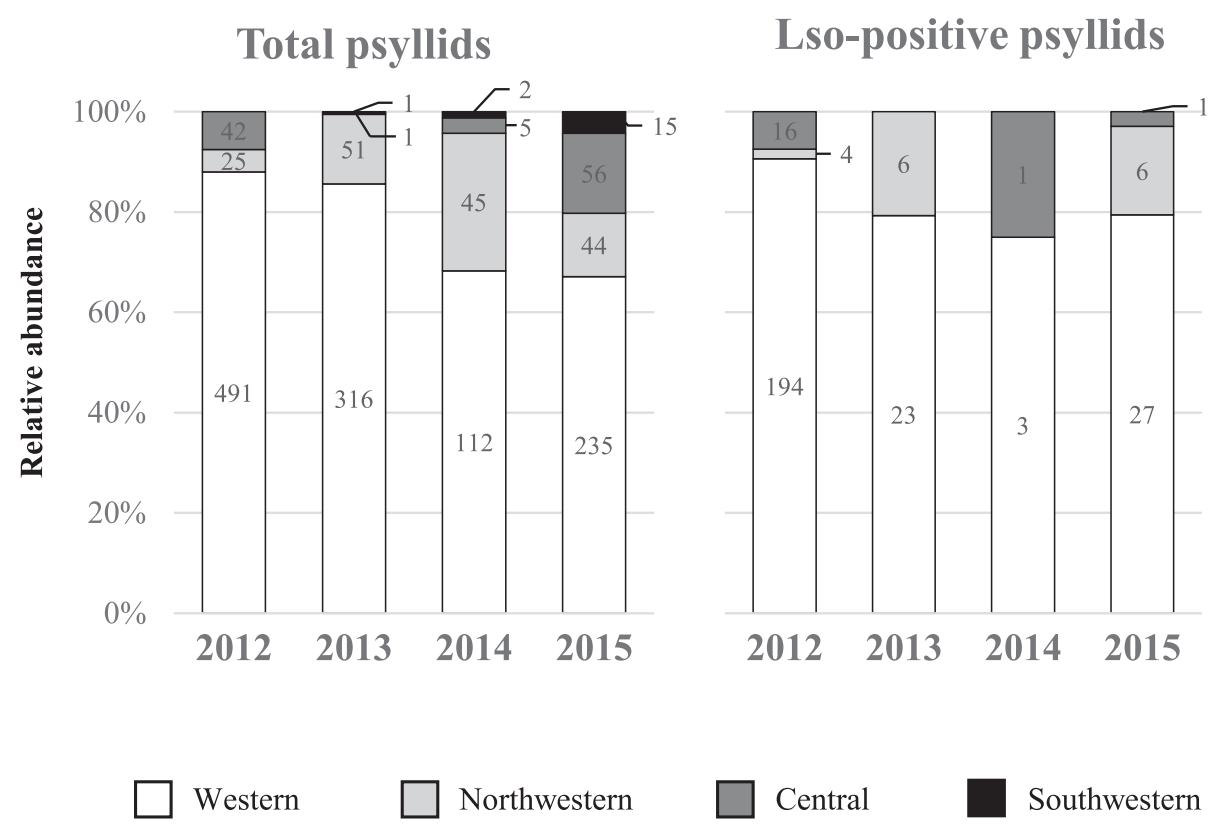

Fig. 4. Analysis of the population of haplotyped psyllids during the potato growing seasons of 2012 to 2015 . A subset of Lso-negative psyllids and all the Lso-positive psyllids, for each year, were subjected to High Resolution Melting analysis for haplotyping. The relative abundance of each haplotype is reported for each year. For Lso-positive psyllids, actual numbers of psyllids for each haplotype is reported on the figure.

2012

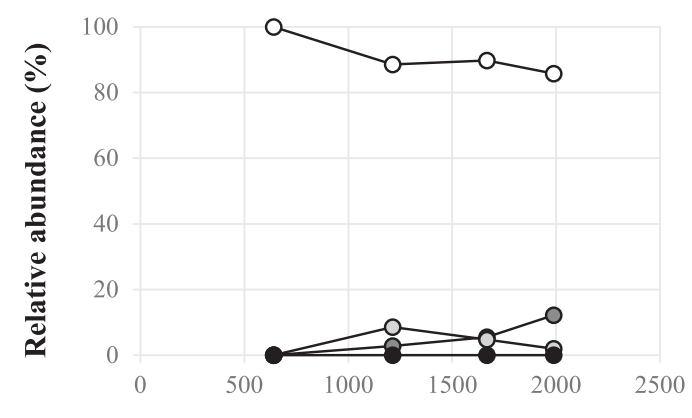

Cumulative degree-days

2014

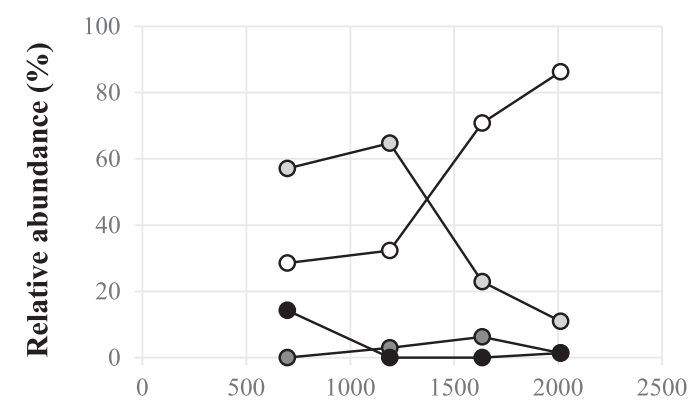

Cumulative degree-days
2013

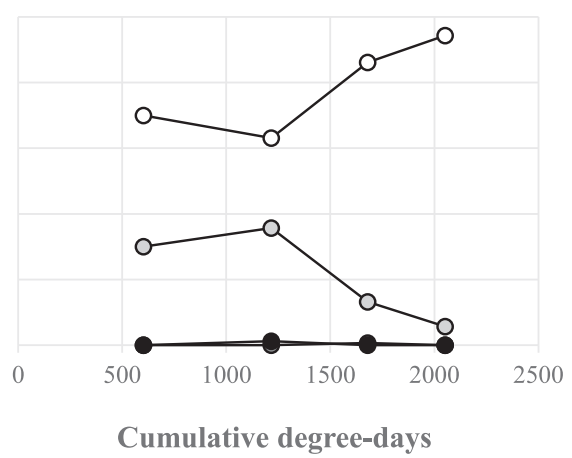

2015

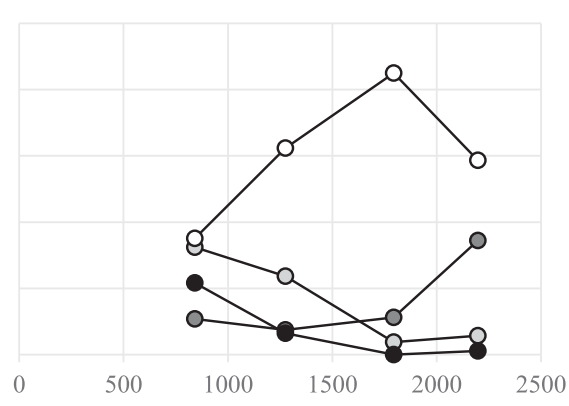

Cumulative degree-days

$$
\text { - }- \text { Western } \quad-\text { - Northwestern }-0 \text { Central } \rightarrow \text { Southwestern }
$$

Fig. 5. Temporal analysis of the population of haplotyped psyllids during each potato growing season. The relative abundance of each psyllid haplotype (obtained by HRM analysis) was analyzed each month of the potato growing seasons, from June to September, for each year from 2012 to 2015 , and plotted against the cumulative degree-days at the end of each month. 
an increasing diversity among the years, with a very mixed population in 2015.

Psyllid haplotypes relative abundances are different depending on location. Based in part on population centers and on different agricultural practices, the Snake River Basin in southern Idaho can be divided into at least two large areas: the Magic Valley and the Treasure Valley (Fig. 1). Numbers of collected and haplotyped psyllids, for each monitored county, are presented in Supplementary Table S2. For each year for which geographical information was available, psyllids were the most abundant in the Treasure Valley, representing over $65 \%$ of the samples. In this area, Ada and Canyon counties were accountable for the majority of them. In the Magic Valley, most psyllids were found in Twin Falls County in 2013 and 2014, whereas in 2015, Cassia and Gooding counties accounted for the majority of psyllids.

The Treasure and Magic valleys also exhibited a different composition of psyllids, with some changes over the years (Fig. 1). Data from 2012 were not available. It appeared that Western psyllids comprised most of the psyllid population in the Treasure Valley, with a relative abundance of $\sim 92 \%$ in 2013 and close to $80 \%$ in 2014 and 2015. Northwestern and Central psyllids represented the remainder. While the Northwestern population remained quite stable from 2013 to 2015 , the relative abundance of Central psyllids steadily increased, representing $0.44 \%, 4.2 \%$, and $12.8 \%$ in 2013,2014 , and 2015 , respectively.

In the Magic Valley, the psyllid population was found to be more evenly mixed. The population was mostly composed of Western and Northwestern haplotypes in 2013 and 2014. Western psyllid abundance was $62.5 \%$ and $35.7 \%$ in those years, respectively, while Northwestern psyllid abundance was $36.5 \%$ and $59.5 \%$ in the same years, respectively. Relative abundance of Southwestern psyllids showed a slight increase over time in the Magic Valley, from $0.9 \%$ in 2013 to $8.5 \%$ in 2015. In 2015, Northwestern psyllid abundance decreased to $26.2 \%$ and Western psyllid abundance remained stable at $43.2 \%$. A sudden rise in the Central haplotype, absent in 2013 and 2104, was observed in 2015, reaching 22\%. The Magic Valley appeared to have a very mixed population of psyllid haplotypes in 2015, as compared with the Treasure Valley, with all four haplotypes represented, while Western psyllid haplotype remained the dominant one in the Treasure Valley.

Discrepancies in psyllid haplotype sequences have been detected in the field in 2015. The CO1 fragment used for haplotyping the psyllids (Swisher et al. 2012) was systematically sequenced for Lso-positive psyllids since 2013, and the amplified product from the Lso-negative psyllids was sequenced when HRM analysis was inconclusive. In 2013 and 2014, the sequences of this fragment for each sample were identical to the published sequences for the known haplotypes. However, in 2015, the sequencing revealed deviations from the published sequences for six Lso-positive and two Lso-negative samples (among the 82 sequenced in 2015) for the CO1 region studied (Supplemental Fig. S1). These psyllids harboring the deviating sequences were collected mainly in Ada and Canyon counties (Treasure Valley), but also in Gooding and Minidoka counties (Magic Valley), in late June and through July. Four of these psyllids harbored a unique SNP in their respective sequence, which is not found in other known haplotypes. The other four had a sequence identical to the Southwestern haplotype for the $5^{\prime}$ terminal of the CO1 fragment, while the $3^{\prime}$ portion was identical to the Western haplotype (Table 2).

\section{Discussion}

In this study, we took advantage of a multiyear monitoring project of potato psyllids in southern Idaho in order to investigate the dynamics of their populations with regard to the described haplotypes, and to examine the haplotypes of Lso found in positive samples during the four years of observation. This large dataset allowed us to gain insights into the spatiotemporal dynamics of psyllids and to speculate on Lso presence in southern Idaho.

To quickly haplotype the Lso carried by psyllids, a method based on a CAPS marker was developed. This method could also be applied successfully in tubers of infected potato plants (data not shown). Using that method, it was determined that in 2012 all of the haplotyped Lso-positive samples exhibited hapA, confirming a previous analysis of a subset of the same Idaho Lso-positive psyllids collection (Swisher et al. 2014). However, by 2013 and onward, hapB Lso appeared and increased in relative abundance. This expansion of the hapB abundance seems to support the hypothesis that psyllids invaded southern Idaho fields, have come northward from southern regions, where hapB used to be predominant. Interestingly, a reverse situation was observed in Texas from 2008 to 2012, where hapB Lso abundance decreased in potato plants and in captured potato psyllids (Wen et al. 2013). A more deleterious effect of hapB Lso than of hapA on bacterium-carrying psyllids was suggested to explain this inversion (Wen et al. 2013), but our results are not consistent with this idea. In addition, the persistence and increase of the hapB Lso abundance in Idaho-caught psyllids after the 2012 season could also suggest that hapB-Lso infected psyllids were able to overwinter in the Pacific Northwest. However, to the best of our knowledge no data are available regarding any infectivity, pathogenicity, or persistence differences between these two haplotypes of Lso that could explain changes in Lso population.

In 2015 we sampled two psyllids carrying both hapA and hapB Lso. A previous Lso haplotyping study showed a percentage from 10 to $100 \%$ of Lso-positive psyllids with coinfection in archived samples from Washington in 2010, Mexico in 2011, and Texas in 2011 and 2012 (Wen et al. 2013). Our results confirm that coinfection can occur; however, questions regarding prevalence and incidence of coinfection, transmission efficiency, and any interactive effects between haplotypes remain open and will require more studies.

Overall, the field population of potato psyllids in southern Idaho was found to be largely dominated by the Western haplotype, corroborating previous data (Swisher et al. 2012, 2014). The notable presence of the Central haplotype was also confirmed, along the Northwestern haplotype. Unexpectedly though, an increasing abundance of Southwestern psyllids was revealed over the years. As it was already hypothesized by Swisher et al. (2014), these data confirm Idaho as a melting-pot for the different potato psyllid haplotypes, especially in the Magic Valley. Several hypotheses can be suggested to explain this fact. This could be due to wind currents bringing psyllids from more diverse geographic areas than the ones moving psyllids into the Columbia basin and Treasure Valley. It has been hypothesized that psyllids could reach the Pacific Northwest from southern regions along two major migratory routes: one roughly along the Pacific coast area and another following the Rocky Mountains (Horton et al. 2015). The difference in crop cultures and the presence of alternative host plants might also be a driving factor accentuating the differences between Treasure Valley and Magic Valley.

Fluctuations in the abundance of psyllid populations was documented over four consecutive potato growing seasons. While populations of the Western haplotype was fairly stable, populations of

Table 2. Data on the psyllids harboring a divergent CO1 fragment sequences, collected during the 2015 potato growing season

\begin{tabular}{lclcc}
\hline & Collection Date $^{\mathbf{a}}$ & \multicolumn{1}{c}{ Site $^{\mathbf{b}}$} & Lso $^{\mathbf{c}}$ & Mutation $^{\mathbf{d}}$ \\
\hline Mut1 & Jun. 30 & Canyon & - & $\mathrm{SW} / \mathrm{W}$ \\
Mut2 & Jul. 6 & Canyon & $+(\mathrm{B})$ & $\mathrm{SW} / \mathrm{W}$ \\
Mut3 & Jul. 6 & Ada & $+(\mathrm{B})$ & $\mathrm{SW} / \mathrm{W}$ \\
Mut4 & Jul. 7 & Minidoka & - & $\mathrm{SW} / \mathrm{W}$ \\
Mut5 & Jul. 20 & Ada & $+(\mathrm{B})$ & $\mathrm{W}$ \\
Mut6 & Jul. 20 & Ada & $+(\mathrm{B})$ & $\mathrm{C}$ \\
Mut7 & Jul. 20 & Ada & $+(\mathrm{B})$ & $\mathrm{C}$ \\
Mut8 & Aug. 19 & Gooding & $+(\mathrm{B})$ & $\mathrm{W}$ \\
\hline
\end{tabular}

a The date on which the sticky cards where the psyllids were caught were recovered from the field.

$\mathrm{b}$ The county where the psyllid was collected.

${ }^{\mathrm{c}}$ Indicates if the psyllid was positive (+) or negative (-) for Lso. In case of positivity, the Lso haplotype is indicated in parentheses (ND, not determined).

${ }^{d}$ The type of mutation identified in the sequenced $\mathrm{CO} 1$ fragment, as presented in Supplemental Fig. S2. SW/W, mixed fragment of Southwestern and Western haplotype; Point mutation indicates a SNP in the given background (W, western; C, central). 
Central, Northwestern, and Southwestern haplotypes were shown to be more dynamic. The absence of Central psyllids in 2013 among the haplotyped subset was notable; it reappeared at a low level in 2014, before increasing in abundance in 2015. Similarly, Southwestern psyllids, absent in 2012 and 2013, appeared in 2014 and persisted in the fields in 2015. Central, Western, and Southwestern haplotypes are probably not endogenous haplotypes of the Pacific Northwest, as opposed to Northwestern psyllids. Infestations of psyllids in the Pacific Northwest fields have been well documented (Butler and Trumble 2012; Horton et al. 2015; Nelson et al. 2014), and it is now assumed that presence of Western, Central, and Southwestern psyllids in the Pacific Northwest is most likely due to the migration of those psyllids from their respective origins (Munyaneza et al. 2009). These seasonal migrations have been proposed to result from local temperature elevations in the southernmost regions, less favorable to psyllids development which in turn move northward to cooler places. While the presence of all four psyllid haplotypes in Idaho potato fields can be explained this way, this does not clarify the observed trends beginning in 2014, where Western and Northwestern psyllids decreased in abundance relative to Central and Southwestern ones.

A seasonal trend in the population of Northwestern psyllids was also revealed, increasing in the fields at the beginning of the season before collapsing. It has been suggested that since the Northwestern haplotype has not yet been found outside the Pacific Northwest region, its presence each year in southern Idaho fields is likely an indicator of its ability to overwinter on local host plants (Horton et al. 2015; Murphy et al. 2014; Swisher et al. 2013b). This overwintering capacity has long been associated with cold hardiness. A recent monitoring study in Washington has failed to find any other type of psyllids besides Northwestern on one of the local overwintering hosts, Solanum dulcamara (bittersweet nightshade) (Swisher et al. 2013b). Furthermore, greenhouse and field studies have shown that at least Central psyllids can also survive cold temperatures (Henne et al. 2010; Horton et al. 2014; Whipple et al. 2012). It has been shown that all haplotypes could feed and reproduce on this host (Mustafa et al. 2015a), strongly suggesting that it could well be possible for the psyllids to shift to this plant at the end of the potato growing seasons, given favorable conditions. In that case, this could in part explain why there was an increase in Southwestern and Central psyllids in 2015, after their settlement on local overwintering host plant in 2014. Indeed, locally overwintering psyllids would then have easier and earlier access to potato fields as compared from migrating ones.

In regard to ZC epidemiology, any relationships between psyllid haplotypes and Lso infection are unclear at this point (Mustafa et al. 2015b). All psyllid haplotypes are able to carry Lso and to infect potato plants, and haplotype A and B Lso have been found in Central, Northwestern, and Western psyllids, indicating that all psyllid haplotypes are able to carry both Lso haplotypes.

The Snake River Basin in South Idaho is traditionally divided into two major agricultural areas: the Magic Valley, near Twin Falls; and the Treasure Valley, around Boise. As a clear difference in psyllid infestations in the 2012 growing season was revealed between these two regions (Wenninger et al. 2013), psyllids collected from fields located in these two areas were analyzed separately. Clear differences could be observed in spatial as well as temporal patterns of psyllid populations within each valley. In particular, changes of psyllid abundance are more noticeable in the Magic Valley over the four years of observation, with an increasing abundance of Central and Southwestern haplotypes, concomitant with the reduced abundance of the Western and Northwestern psyllids. This suggests that changes in psyllid population composition occur mainly in the Magic Valley. Several hypotheses could explain these changes. First, the Magic Valley may be an entry point of migratory psyllids coming from areas where southcentral psyllids are dominant; they would then settle first in that area, before moving further toward the Treasure Valley at the end of the season, explaining the lower changes in haplotypes composition there. Second, the Magic Valley could also be an overwintering spot, with more opportunities for psyllids to accommodate after the end of the potato growing season (such as presence of alternative host(s), meteorological conditions). Third, psyllids coming from southern regions could have better conditions for reproduction in the Magic Valley than in the Treasure Valley, explaining a better representation in the Magic Valley.

Within a subset of 2015 Lso-positive and Lso-negative samples, several were found to have mutations in the mitochondrial CO1 pseudogene fragment. To our knowledge, this is the first report of divergent sequences for established haplotypes. No data are presently available about mutation rate in psyllids, but this is an indication of evolution of Central and Western psyllids, changing their CO1-based haplotype assignments. Furthermore, the presence of psyllids with "mixed" CO1 sequences between Western and Southwestern haplotypes strongly suggests that this mutation occurred in Idaho. Indeed, Western and Southwestern haplotypes populate different and geographically unrelated areas, and likewise have different migratory routes to access Idaho fields.

Another interesting observation is that relative to the number of sequenced samples, it seems that the mutants were more abundant in the Lso-positive samples, and only with hapB Lso. This raises the question of a possible coevolution of psyllids with the bacterium, perhaps influencing psyllid fitness in the presence of the parasite.

This study demonstrated that the psyllid populations in Southern Idaho potato fields are highly dynamic over time and space. Moreover, the Lso population, which depends on the movements of its insect vector, was found to change over time, even though disease pressure remained low in the fields. Complicating the situation is the evidence of increasing diversity among the existing haplotypes, probably due to mating. While there were no connections found between the haplotypes of either Lso or the psyllids and the probability of the ZC disease outbreak, more observations of both vector and pathogen populations are needed to determine the major factors accounting for Lso and $\mathrm{ZC}$ disease outbreaks.

\section{Acknowledgments}

We are grateful to Alicia Hodnik, Melena Suliteanu, Lauren Merlino, and Eliza Clark for help in sample processing. For assistance with managing the potato psyllid monitoring network, we thank: Amy Carroll, Lucy Standley, Lynn Woodell, Mike Thornton, Phil Nolte, Oksana Adams, Melissa Bertram, Jill Randall, Melinda Lent, Jennifer Riebe, Tom Salaiz, Drew Glascock, Jeff Miller, Katherine Long, Megan Williams, Paul Stukenholtz, Janan Claiborn, Dusty Danos, Jessica Vogt, Anastasia Stanzak, Cheryn Clayton, Neyle Perdomo, Vince Adamson, Tucker Daley, Kortni Cox, Aaron Vogt, Carlie Wilkinson, Jesica Lowe, Kyanne Frandsen, Chelsea Stevens, Ethan Whitten, Kevin Robison, Wyatt Shewmaker, Austin Fife, and Trent Taysom. This work was funded in part through grants from Idaho State Department of Agriculture, USDA-ARS (58-5354-2-345 and 58-5352-4-006),

USDA-TASC, Northwest Potato Research Consortium, Idaho Potato Commission, and by the Idaho Agricultural Experiment Station.

\section{Literature Cited}

Ananthakrishnan, G., Choudhary, N., Roy, A., Sengoda, V. G., Postnikova, E., Hartung, J. S., Stone, A. L., Damsteegt, V. D., Schneider, W. L., Munyaneza, J. E., and Brlansky, R. H. 2013. Development of primers and probes for genus and species specific detection of "Candidatus Liberibacter species" by real-time PCR. Plant Dis. 97:1235-1243.

Butler, C. D., and Trumble, J. T. 2012. Identification and impact of natural enemies of Bactericera cockerelli (Hemiptera: Triozidae) in southern California. J. Econ. Entomol. 105:1509-1519.

Chapman, R. I., Macias-Velasco, J. F., Arp, A. P., and Bextine, B. R. 2012. Using quantitative real time pcr melt curve analysis of partial CO1 sequence for rapid biotype differentiation of Bactericera cockerelli (Hemiptera: Triozidae). Southwest. Entomol. 37:475-484.

Crosslin, J. M., Hamm, P. B., Eggers, J. E., Rondon, S. I., Sengoda, V. G., and Munyaneza, J. E. 2012a. First report of Zebra Chip disease and "Candidatus Liberibacter solanacearum" on potatoes in Oregon and Washington state. Plant Dis. 96:452-453.

Crosslin, J. M., Lin, H., and Munyaneza, J. E. 2011. Detection of "Candidatus Liberibacter solanacearum" in the potato psyllid, Bactericera cockerelli (Sulc), by conventional and real-time pcr. Southwest. Entomol. 36:125-135.

Crosslin, J. M., Olsen, N., and Nolte, P. 2012b. First report of Zebra Chip disease and "Candidatus Liberibacter solanacearum" on potatoes in Idaho. Plant Dis. 96:453.

Goolsby, J. A., Adamczyk, J. J., Crosslin, J. M., Troxclair, N. N., Anciso, J. R., Bester, G. G., Bradshaw, J. D., Bynum, E. D., Carpio, L. A., Henne, D. C., Joshi, A., Munyaneza, J. E., Porter, P., Sloderbeck, P. E., Supak, J. R., Rush, C. M., Willett, F. J., Zechmann, B. J., and Zens, B. A. 2012. Seasonal population dynamics of the potato psyllid (Hemiptera: Triozidae) and its associated pathogen "Candidatus Liberibacter solanacearum" in potatoes in the southern great plains of North America. J. Econ. Entomol. 105:1268-1276.

Hansen, A. K., Trumble, J. T., Stouthamer, R., and Paine, T. D. 2008. A new Huanglongbing species, "Candidatus Liberibacter psyllaurous", found to infect 
tomato and potato, is vectored by the psyllid Bactericera cockerelli (Sulc). Appl. Environ. Microbiol. 74:5862-5865.

Henne, D. C., Workneh, F., and Rush, C. M. 2010. Movement of Bactericera cockerelli (Heteroptera: Psyllidae) in relation to potato canopy structure, and effects on potato tuber weights. J. Econ. Entomol. 103:1524-1530.

Horton, D. R., Cooper, W. R., Munyaneza, J. E., Swisher, K. D., Echegaray, E. R., Murphy, A. F., Rondon, S. I., Wohleb, C. H., Waters, T. D., and Jensen, A. S. 2015. A new problem and old questions: potato psyllid in the Pacific Northwest. Am. Entomol. 61:234-244.

Horton, D. R., Munyaneza, J. E., Swisher, K. D., Echegaray, E. R., Murphy, A. F., Rondon, S. I., Sengoda, V. G., Neven, L. G., and Jensen, A. S. 2014. What is the source of potato psyllids colonizing Washington, Oregon, and Idaho potato fields? Potato Prog. 14:1-6.

Liefting, L. W., Sutherland, P. W., Ward, L. I., Paice, K. L., Weir, B. S., and Clover, G. R. G. 2009. A new "Candidatus Liberibacter" species associated with diseases of solanaceous crops. Plant Dis. 93:208-214.

Liu, D., Trumble, J. T., and Stouthamer, R. 2006. Genetic differentiation between eastern populations and recent introductions of potato psyllid (Bactericera cockerelli) into western North America. Entomol. Exp. Appl. 118:177-183.

Loiseau, M., Gamier, S., Boirin, V., Merieau, M., Leguay, A., Renaudin, I., Renvoise, J.-P., and Gentit, P. 2014. First report of "Candidatus Liberibacter solanacearum" in carrot in France. Plant Dis. 98:839.

Munyaneza, J. E. 2012. Zebra Chip disease of potato: Biology, epidemiology, and management. Am. J. Potato Res. 89:329-350.

Munyaneza, J. E., Crosslin, J. M., and Buchman, J. L. 2009. Seasonal occurrence and abundance of the potato psyllid, Bactericera cockerelli, in South Central Washington. Am. J. Potato Res. 86:513-518.

Munyaneza, J. E., Fisher, T. W., Sengoda, V. G., Garczynski, S. F., Nissinen, A., and Lemmetty, A. 2010. First report of "Candidatus Liberibacter solanacearum" associated with psyllid-affected carrots in Europe. Plant Dis. 94:639.

Munyaneza, J. E., Swisher, K. D., Hommes, M., Willhauck, A., Buck, H., and Meadow, R. 2015. First report of "Candidatus Liberibacter solanacearum" associated with psyllid-infested carrots in Germany. Plant Dis. 99:1269.

Murphy, A. F., Cating, R. A., Goyer, A., Hamm, P. B., and Rondon, S. I. 2014. First report of natural infection by "Candidatus Liberibacter solanacearum" in bittersweet nightshade (Solanum dulcamara) in the Columbia basin of Eastern Oregon. Plant Dis. 98:1425.

Murphy, A. F., Rondon, S. I., and Jensen, A. S. 2013. First report of potato psyllids, Bactericera cockerelli, overwintering in the Pacific Northwest. Am. J. Potato Res. 90:294-296.

Mustafa, T., Horton, D. R., Cooper, W. R., Swisher, K. D., Zack, R. S., and Munyaneza, J. E. 2015a. Interhaplotype fertility and effects of host plant on reproductive traits of three haplotypes of Bactericera cockerelli (Hemiptera: Triozidae). Environ. Entomol. 44:300-308.

Mustafa, T., Horton, D. R., Cooper, W. R., Swisher, K. D., Zack, R. S., Pappu, H. R., and Munyaneza, J. E. 2015b. Use of electrical penetration graph technology to examine transmission of "Candidatus Liberibacter solanacearum" to potato by three haplotypes of potato psyllid (Bactericera cockerelli; Hemiptera: Triozidae). PLOS ONE doi:10:e0138946

Mustafa, T., Horton, D. R., Swisher, K. D., Zack, R. S., and Munyaneza, J. E. 2015c. Effects of host plant on development and body size of three haplotypes of Bactericera cockerelli (Hemiptera: Triozidae). Environ. Entomol. 44:593-600.

Nelson, W. R., Fisher, T. W., and Munyaneza, J. E. 2011. Haplotypes of "Candidatus Liberibacter solanacearum" suggest long-standing separation. Eur. J. Plant Pathol. 130:5-12.

Nelson, W. R., Sengoda, V. G., Alfaro-Fernandez, A. O., Font, M. I., Crosslin, J. M., and Munyaneza, J. E. 2013. A new haplotype of "Candidatus Liberibacter solanacearum" identified in the Mediterranean region. Eur. J. Plant Pathol. 135:633-639.

Nelson, W. R., Swisher, K. D., Crosslin, J. M., and Munyaneza, J. E. 2014. Seasonal Dispersal of the Potato Psyllid, Bactericera cockerelli, into Potato Crops. Southwest. Entomol. 39:177-186.

Secor, G. A., and Rivera, V. V. 2004. Emerging diseases of cultivated potato and their impact on Latin America. Rev. Latinoam. Papa Suppl. 1:1-8.
Swisher, K. D., Munyaneza, J. E., and Crosslin, J. M. 2012. High resolution melting analysis of the Cytochrome Oxidase I gene identifies three haplotypes of the potato psyllid in the United States. Environ. Entomol. 41:1019-1028.

Swisher, K. D., Munyaneza, J. E., and Crosslin, J. M. 2013a. Temporal and spatial analysis of potato psyllid haplotypes in the United States. Environ. Entomol. 42:381-393.

Swisher, K. D., Sengoda, V. G., Dixon, J., Echegaray, E., Murphy, A. F., Rondon, S. I., Munyaneza, J. E., and Crosslin, J. M. 2013b. Haplotypes of the potato psyllid, Bactericera cockerelli, on the wild host plant, Solanum dulcamara, in the Pacific Northwestern United Utates. Am. J. Potato Res. 90:570-577.

Swisher, K. D., Sengoda, V. G., Dixon, J., Munyaneza, J. E., Murphy, A. F., Rondon, S. I., Thompson, B., Karasev, A. V., Wenninger, E. J., Olsen, N., and Crosslin, J. M. 2014. Assessing potato psyllid haplotypes in potato crops in the Pacific Northwestern United States. Am. J. Potato Res. 91:485-491.

Tahzima, R., Maes, M., Achbani, E. H., Swisher, K. D., Munyaneza, J. E., and De Jonghe, K. 2014. First report of "Candidatus Liberibacter solanacearum" on carrot in Africa. Plant Dis. 98:1426.

Teresani, G. R., Bertolini, E., Alfaro-Fernandez, A., Martínez, C., Tanaka, F. A O., Kitajima, E., Rosello, M., Sanjuan, S., Ferrandiz, J. C., López, M. M. Cambra, M., and Font-San-Ambrosio, M. I. 2014. Association of "Candidatus Liberibacter solanacearum" with a vegetative disorder of celery in Spain and development of a real-time PCR method for its detection. Phytopathology 104:804-811.

Thinakaran, J., Pierson, E., Kunta, M., Munyaneza, J. E., Rush, C. M., and Henne, D. C. 2015b. Silverleaf nightshade (Solanum elaeagnifolium), a reservoir host for "Candidatus Liberibacter solanacearum", the putative causal agent of Zebra chip disease of potato. Plant Dis. 99:910-915.

Thinakaran, J., Pierson, E. A., Longnecker, M., Tamborindeguy, C., Munyaneza, J. E., Rush, C. M., and Henne, D. C. 2015a. Settling and ovipositional behavior of Bactericera cockerelli (Hemiptera: Triozidae) on solanaceous hosts under field and laboratory conditions. J. Econ. Entomol. 108:904-916.

Thinakaran, J., Yang, X.-B., Munyaneza, J. E., Rush, C. M., and Henne, D. C 2015c. Comparative biology and life tables of "Candidatus Liberibacter solanacearum"-infected and -free Bactericera cockerelli (Hemiptera: Triozidae) on potato and silverleaf nightshade. Ann. Entomol. Soc. Am. 108: 459-467.

Thompson, S. M., Johnson, C. P., Lu, A. Y., Frampton, R. A., Sullivan, K. L., Fiers, M. W. E. J., Crowhurst, R. N., Pitman, A. R., Scott, I. A. W., Wen, A. Gudmestad, N. C., and Smith, G. R. 2015. Genomes of "Candidatus Liberibacter solanacearum" haplotype A from New Zealand and the United States suggest significant genome plasticity in the species. Phytopathology 105:863-871.

Tran, L. T., Worner, S. P., Hale, R. J., and Teulon, D. J. 2012. Estimating development rate and thermal requirements of $<i>$ Bactericera cockerelli $<\mathrm{i} />$ (Hemiptera: Triozidae) reared on potato and tomato by using linear and nonlinear models. Environ. Entomol. 41:1190-1198.

Wen, A., Johnson, C., and Gudmestad, N. C. 2013. Development of a PCR assay for the rapid detection and differentiation of "Candidatus Liberibacter solanacearum" haplotypes and their spatiotemporal distribution in the United States. Am. J. Potato Res. 90:229-236.

Wen, A., Lin, H., and Gudmestad, N. C. 2011. Development of PCR assay using simple sequence repeat primers for detection of "Candidatus Liberibacter solanacearum.". Phytopathology 101:S190.

Wenninger, E. J., Olsen, N., Thornton, M., Nolte, P., and Karasev, A. V. 2013 Monitoring of potato psyllids, Candidatus Liberibacter solanacearum, and zebra chip in Idaho during the 2013 growing season. P. of the 12th Annu. SCRI Zebra Chip reporting session, San Antonio, TX.

Whipple, S. D., Bradshaw, J. D., and Harveson, R. M. 2012. Cold tolerance in potato psyllids. P. of the 12th Annu. SCRI Zebra Chip reporting session, San Antonio, TX. 